\section{Preparatory-response vs information-seeking interpretations of preference for signaled loud noise: Further limits on human informational cognitive control*}

\author{
JOHN J. FUREDY, DALE FAINSTAT, PAMELLA KULIN, \\ LISA LASKO, and SANDRA NICHOLS \\ University of Toronto, Toronto 181 , Ont., Canada
}

Fifty-six Ss received a series of .3 -sec noises of varying intensity $(80,90,100$ 110 , or $120 \mathrm{~dB}$ ). Half the noises were preceded by an 8 -sec signal, and a postexperimental questionnaire indicated a reliable group preference for signaled over unsignaled noise. A preparatory-response interpretation of the preference requires the presence of informational cognitive control, defined as occurring, whenever the signal reduces the subjectively rated intensity of a noxious inescapable outcome. No such reduction was observed, even though the subjective intensity ratings were clearly sensitive to physical noise-intensity differences. The results are consistent with an information-seeking interpretation of the preference-for-signaled-noise phenomenon.

It is commonly believed that signaled inescapable noxious outcomes are preferred over the same unsignaled outcomes (e.g., Lanzetta \& Driscoll, 1966). The occurrence of this preference is consistent with, but does not imply, the phenomenon of informational cognitive control, which has been defined by Furedy \& Doob (1972) as occurring whenever the signal reduces the felt aversiveness of the noxious outcome. The mechanism through which such control might operate has been specified recently by Perkins (1968) in terms of the signal-elicited preparatory response (PR), which adaptively prepares $S$ for the noxious outcome by reducing the outcome's psychological impact. An account which differs from this $P R$ interpretation of the signaled preference phenomenon, and which does not imply any informational cognitive control in the sense defined above, is the information-seeking (IS) interpretation of Berlyne (1960). The IS interpretation suggests that the signal is reinforcing (and hence preferred) because it reduces uncertainty rather than actually reducing outcome aversiveness.

Any attempt to reach a decision between the PR and IS accounts of the preference phenomenon, however, has to contend with a more recent and

*The participation of D.F., P.K., L.L., and S.N. in this research was made possible by a grant from the Opportunities for Youth Programme of Canada (300-3575). The laboratory and other technical facilities used for the research were supported from grants to J.J.F. from Canada Council (S70-0710) and the National Research Council of Canada (APA 222). We are indebted to A. N. Doob for some critical advice. theoretically awkward set of findings by Furedy \& Doob (1972). With shock as the noxious inescapable outcome. these investigators were unable to find any evidence for the preference phenomenon itself, whether the preference was measured in terms of postexperimental questionnaires (Furedy \& Doob, 1972, Experiments II and III) or by observations of actual behavior (Furedy \& Doob, 1972, Experiment IV). Moreover, if one accepts Furedy and Doob's arguments for concluding that Lanzetta and Driscoll's "experiment was not optimally designed to test for a preference for signaled over unsignaled shock [Furedy \& Doob, 1972, p. 114]," one is left in the embarrassing situation where a decision between the $P R$ and IS interpretations of the preference phenomenon seems not only difficult to reach, but also hardly worth the trouble, since the phenomenon itself appears not to exist.

However, it is possible that the preference phenomenon may emerge if loud noise rather than shock is the noxious inescapable outcome. In that case, in contrast to the shock situation, there is a specifiable mechanism which could potentially allow the signal to reduce noise aversiveness-the middle-ear reflex (e.g., Fletcher, 1962). Accordingly, the present study was run along the lines of the original shock study reported by Furedy \& Doob (1972, Experiment I), but with noise as the signaled and unsignaled outcome. In addition, as in the later shock studies (Furedy \& Doob, 1972, Experiments II and III), a postexperimental questionnaire assessed the preference for signaled over unsignaled noise. The first aim was to determine if Ss exposed to signaled and unsignaled noise would prefer the former type of noxious inescapable outcome. Secondly, information was obtained on the relative aversiveness of signaled and unsignaled noise, as in the original shock study (for details, ef. Furedy, 1970), by having Ss rate the intensity of each presented noise. Moreover, the validity of these rated-intensity (RI) indices was checked within the experiment, as in the original shock study, by seeing whether RI was reliably sensitive to physical intensity differences. Then, if both the preference occurred and the $R I$ index was shown to be valid, the intention was to arbitrate between the PR and IS interpretations of the preference phenomenon by seeing whether signaling did reduce the felt aversiveness of the inescapable noise outcome.

The Ss were 56 undergraduates from the University of Toronto, paid $\$ 1.50$ to participate. The apparatus was the same as that detailed previously (Furedy, 1970), except that the white-noise stimulus, which originated from a Grason-Stadler generator, was presented to $S$ for $.3 \mathrm{sec}$ through earphones and was variable in $10-\mathrm{dB}$ steps from 80 to $120 \mathrm{~dB}$ SPL. Subjectively, it is at least clear that the $120-\mathrm{dB}$ noise was quite aversive, as indicated by the fact that the laboratory personnel generally refused to tolerate bursts exceeding $.5 \mathrm{sec}$.

The procedure, as in Furedy (1970), comprised a set of differential conditioning trials, with counterbalanced tone and light as the CSs always (CS+) and never (CS-) paired with the (white-noise) UCS. In addition to the CS + UCS (signaled noise) and CS-alone trials, the Ss also received UCS-alone (unsignaled noise) trials, and the intertrial intervals varied unsystematically between 30,40 , and $50 \mathrm{sec}$. The CS-UCS interval and CS duration were both 8 sec. The noise RI measure was obtained by S's rotating a lever up to 180 deg (full scale) with his right hand after the delivery of each noise; lever rotation was registered on a voltmeter in E's room (Furedy, 1970)

At the end of the preliminary stage, where five UCS-alone (increasing from 80 to $120 \mathrm{~dB}$ ) trials were interspersed with two each of the CS+ and CStrials, as in Furedy (1970), the $\mathrm{S}$ was encouraged to use most of the $180 \mathrm{deg}$ of lever rotation by being told that no stronger noises would be presented in the second stage. This stage was identical to that of Furedy (1970) in terms of $\mathrm{CS}+\mathrm{UCS}, \mathrm{CS}-$, and 


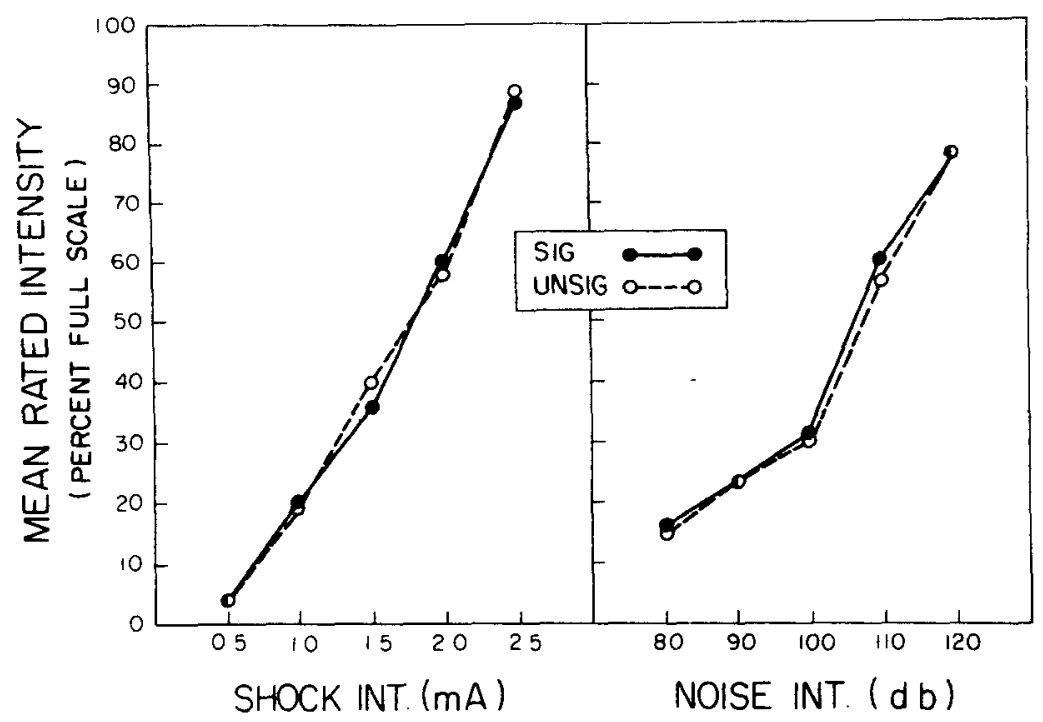

Fig. 1. Mean rated intensity (RI) as a function of signaling and physical-intensity factors with shock (Furedy, 1970) and noise (present study) outcomes. (Because of apparatus failure, the 110-dB noise was not delivered for $18 \mathrm{Ss}$, so that the mean RI values for signaled and unsignaled $110 \mathrm{~dB}$ outcomes are based on only $38 \mathrm{Ss}$.)

UCS-alone trials: $S$ received 15 of each type of trial, and the nature of the trials (including variations in intensity) were unsystematically ordered. Finally, at the end of the experiment, Ss were asked to indicate on a questionnaire whether they preferred signaled noise, unsignaled noise, or had no preference.

\section{RESULTS}

There was a significant, $\mathrm{p}<.02$, preference for signaled noise with 28 , 12 , and 16 Ss expressing signaled, unsignaled, and no preference, respectively. As indicated by the right panel of Fig. 1, the RI index was validated by being reliably sensitive to physical noise-intensity differences, p $<.001$, as was the RI to shock-intensity differences in Furedy's (1970) study (Fig. 1, left panel). However, as in the shock study, the present results also failed to yield any reliable differences in $\mathrm{RI}$ between signaled and unsignaled outcomes at any of the five physical-intensity levels of the inescapable noise outcome used here, ts $<1$.

\section{DISCUSSION}

Although there was a considerable proportion (.29) of Ss who indicated no preference, the remaining group of Ss who did express a preference indicated a marked preference for signaled over unsignaled noise. This reliable group preference for signaling contrasts with the repeated failures of Furedy \& Doob (1972), with shock as the inescapable outcome, to find the preference phenomenon over a considerable range of conditions. Since the present study did not include a concurrently run group of Ss who received the shock rather than sound outcome, the possibility cannot entirely be ruled out that the apparent preference reflects only a difference in $S$ populations. Nevertheless, it is worth noting that if the more likely shock-noise interpretation is favored over the population-difference interpretation, there is a plausible way of accounting for the emergence of the preference with a shift from shock to noise outcomes. That account relates to the role of the middle-ear reflex (e.g., Fletcher, 1962) in providing a source of additional signaled-outcome modification in the case of sound. On this view, the preference for signaled sound phenomenon would be explained in terms of the PR interpretation of Perkins (1966) rather than the IS interpretation of Berlyne (1960). However, the present RI results, where a validated form of the RI measure failed to show any reduction of noise aversiveness as a function of signaling at any of the five physical noise intensity levels, clearly favor the IS over the PR interpretation.

In contrast to our RI results, Grings (1969) has briefly reported that signaling reduced the rated intensity of loud noise, since, in a between-groups design, the intensity of ratings of signaled noises were clearly (Grings, 1969 , Fig. 4) and reliably lower than those of unsignaled noises. However, the results reported by Grings are based on RI measures which were expressed as ratios of later trial to initial trial ratings. A more detailed report of the data in nonratio form by shock-noise difference in signaled
Schell \& Grings (1971, Fig. 2) shows a marked (though reportedly nonsignificant) initial-trial difference in $\mathrm{RI}$, with the signaled noise being rated as more intense than the unsignaled noise. To the extent, then, that the ratio transformation was responsible for producing the signaled $<$ unsignaled $\mathrm{RI}$ results reported by Grings (1969, Fig. 4), those noise RI data do not provide unequivocal evidence for the claim that signaling reduces noise intensity.

Another possibility is that a reduction in $R I$ through signaling would have been obtained in the present instance if a between- rather than a within-Ss design were used. However, at least in the case of shock outcomes, there is evidence against this possibility, since Furedy \& Chan (1971) failed to obtain any reliable reduction in a between-Ss design wherein two groups of $30 \mathrm{Ss}$ were compared. A final consideration is the possibility that the present method of validating the RI index-varying physical noise intensity within Ss-may have resulted in Ss' failing to attend to the finer differences between signaled and unsignaled noises. However, the shock study of Furedy \& Doob (1972, Experiment II) provides at least indirect evidence against this possibility, for in that study shock intensity was held constant between Ss and the signaled-unsignaled RI difference still did not emerge.

All these considerations, of course, are far from sufficient for settling the argument about the effects of signaling noise on noise aversiveness. However, it does bear emphasis that the present noise study did yield a reliable preference for signaled noise and that the $R I$ results favored the IS over the $P R$ interpretation of this preference. At this point, therefore, it seems that, although the shift from shock to noise outcomes has apparently produced evidence for the preference phenomenon, the shift has still not provided the notion of human informational cognitive control with any empirical basis of support.

\section{REFERENCES}

BERLY NE, D. E Conflict, arousal, and curiosity. New York: McGraw-Hill, 1960. FLETCHER, J. L. Reflex response of middle-ear muscles: Protection of the ear from noise. Sound, 1962, 1, 17-23.

FUREDY, J.J. Test of the preparatory-adaptive-response interpretation of aversive classical a utonomic conditioning. Journal of Experimental Psychology, 1970, 84, 301-307.

FUREDY, J. J., \& CHAN, R. M. Failures of information to reduce rated aversiveness of unmodifiable shock. Australian Journal of Psychology, 1971, 23, 85-94. FUREDY, J. J., \& DOOB, A. N. Signaling unmodifial le shocks: Limits on human informational cognitive control. Journal 
of Personality \& Social Psychology, 1972, 21, 111-115.

GRINGS, W. W. Anticipatory and preparatory electrodermal behavior in paired stimulation situations. Psychophysiology, 1969, 5, 597-611.
LANZETT A, J. T., \& DRISCOLL, J. M Preference for information about an uncertain but unavoidable outcome. Journal of Personality \& Social Psychology, 1966, 3, 96-102.

PERKINS, C. C., JR. An analysis of the concept of reinforcement. Psychological Review, 1968, 75, 155-172.

SCHELL, A. M., \& GRINGS, W. W, Judgments of UCS intensity and diminution of the unconditioned GSR. Psychophysiology, 1971, 8, 427-432. 